\title{
BMJ Open Home-based Anti-Tuberculosis Treatment Adverse Reactions (HATTAR) study: a protocol for a prospective observational study
}

\author{
Miaomiao Yang, ${ }^{1}$ Hongqiu Pan, ${ }^{2}$ Lihuan $\mathrm{Lu}^{3}$ Xiaomin $\mathrm{He},{ }^{4}$ Hongbo Chen, ${ }^{5}$ \\ Bilin Tao, ${ }^{1}$ Wenpei Liu, ${ }^{1}$ Honggang Yi, ${ }^{6}$ Shaowen Tang ${ }^{\circ}$
}

To cite: Yang M, Pan H, Lu L, et al. Home-based AntiTuberculosis Treatment Adverse Reactions (HATTAR) study: a protocol for a prospective observational study. BMJ Open 2019;9:e027321. doi:10.1136/ bmjopen-2018-027321

- Prepublication history for this paper is available online. To view these files, please visit the journal online (http://dx.doi org/10.1136/bmjopen-2018027321).

MY and HP contributed equally.

Received 16 October 2018 Revised 11 February 2019 Accepted 27 February 2019

Check for updates

(c) Author(s) (or their employer(s)) 2019. Re-use permitted under CC BY-NC. No commercial re-use. See rights and permissions. Published by BMJ.

For numbered affiliations see end of article.

Correspondence to

Associate Professor Shaowen Tang; tomswen@njmu.edu.cn

\section{ABSTRACT}

Introduction Tuberculosis (TB) continues to be an important public health problem throughout much of the world. Drug treatment is the only effective treatment method, but adverse drug events (ADEs) and adverse drug reactions (ADRs) can affect medication adherence. As the number of drug-resistant TB patients and the number of anti-TB drugs have increased, it is necessary to explore the risk factors for ADEs/ADRs to reduce their occurrence. This study aims to build a home-based anti-TB treatment cohort and to recognise the incidences, prognosis and risk factors of anti-TB drug-induced ADEs/ADRs in real-world experiences.

Methods and analysis This study is a multicentre, prospective observational cohort study. The study population will consist of 3200 newly diagnosed TB patients between January 2019 and December 2020. After initiating the anti-TB treatment, all patients will be followed up until finishing treatment unless they withdraw, and we will record personal drug use and signs and/or symptoms of discomfort. Patients will receive scheduled laboratory tests in designated hospitals every 2 weeks during the first 2 months, and the residual blood sample after conducting the laboratory tests will be preserved. The ADEs/ADRs will be placed into eight categories: liver dysfunction, gastrointestinal reactions, drug allergy, arthralgia or muscle pain, nervous system disorders, haematological system disorders, renal impairment and others.

Ethics and dissemination This study protocol has been approved by the ethics committees of Nanjing Medical University. All patients will give written informed consent before enrollment. The findings of the study will be published in peer-reviewed journals and will be presented at national and international conferences.

\section{INTRODUCTION}

Tuberculosis (TB) is one of the top 10 causes of death and the leading cause from a single infectious agent. In 2017, approximately 10.0 million people developed TB disease, and TB caused an estimated 1.3 million deaths. ${ }^{1}$ Drug treatment is the only effective treatment method for $\mathrm{TB}$, and the WHO has implemented a standardised directly observed

\section{Strengths and limitations of this study}

- This is a prospective study design with a homebased, large-scale consecutive anti-tuberculosis treatment cohort under real-world experiences in China.

- A method combining active self-recorded diaries and passive scheduled laboratory tests will be used to enhance the identification of adverse reactions.

- The residual blood sample after the laboratory tests will be preserved for future research.

- A limitation is that it will recruit the potential eligible patients, including a floating population, who will add uncertainty to the follow-up.

treatment, the short-course (DOTS)/Stop TB Strategy, to improve TB prevention and control. Although the WHO set the global target rate for a successful treatment outcome at $85 \%,{ }^{2}$ the treatment success rate remains low, at $82 \%$ for all TB patients and $55 \%$ for drug-resistant TB (DR-TB) patients. ${ }^{1}$ Many factors have influenced the treatment success rate, such as sociodemographic and socioeconomic factors, nutrition, HIV, drug resistance and strategies for TB management including DOTS. ${ }^{3}$ Additionally, effective chemotherapy is largely based on patients' willingness to comply with the prescribed regimen, and adverse drug events (ADEs) and adverse drug reactions (ADRs) are significant factors affecting the adherence of patients to medications. ${ }^{4}$ According to WHO, an ADE is defined as any untoward medical occurrence that may appear during treatment with a pharmaceutical product but which does not necessarily have a causal relationship with the treatment ${ }^{5}$; and an ADR is any response to a drug that is noxious and unintended and that occurs at doses normally used in humans for prophylaxis, diagnosis or therapy of disease, or for the modification of physiological function. ${ }^{6}$ 
Long-term anti-TB combination regimens could lead to various types and levels of ADEs/ADRs, which could subsequently lead to treatment discontinuation or interruption, treatment time extension and an increased risk for developing drug resistance, treatment failure and relapse. ${ }^{7}$ All of these negative consequences have posed a challenge to TB treatment and have implications for TB control.

Among the various ADEs/ADRs, the most common ones induced by first-line anti-TB drugs are hepatotoxicity, gastrointestinal disorders, allergic reactions, arthralgia, neurological disorders, ${ }^{8}$ while in multidrug-resistant TB (MDR-TB) patients, common types are arthralgia, gastrointestinal disorders, hypothyroidism, dermatologic disorders, haematological disorders, hepatotoxicity, ototoxicity and nervous system or psychiatric disorders. ${ }^{9}$ TB patients treated by second-line anti-TB drugs in the context of high HIV coinfection had more severe ADEs/ADRs, and the most common severe type reported was hearing loss or ototoxicity. ${ }^{10}$ Women, patients of an advanced age and recurrent TB patients were found to be at a higher risk of leucopenia. ${ }^{11}$ As problems such as DR-TB, MDR-TB, extensively drug-resistant TB (XDR-TB), and a recently emerging threat by a totally drug-resistant TB (TDR-TB) are increasing in prevalence, several novel drugs will cause a significant shift in the landscape of TB treatment but can cause some other ADEs/ADRs. For example, bedaquiline and delamanid can prolong cardiac QTc. ${ }^{12} 13$ The majority of TB patients receive community-based or home-based treatment; however, most studies of ADRs are hospital-based..$^{9-11}$ Treatment interruption due to ADEs/ADRs could decrease the chance of a cure ${ }^{14}$ and lead to patient loss to follow-up, further increasing the development of drug resistance. ${ }^{15}$ Additionally, community-based care for MDR-TB patients is more effective than care in a central specialised hospital. ${ }^{16}$ Home-based care could increase MDR-TB treatment success ${ }^{16}$ and has potential in improving treatment outcomes. ${ }^{17}$ Therefore, it is necessary to strengthen research on ADEs/ADRs in home-based anti-TB treatment.

Between October 2007 and June 2008, a cohort of Anti-tuberculosis Drugs induced Adverse Reactions in China National Tuberculosis Prevention and Control Scheme Study (ADACS) was established by us in China. ${ }^{18}$ Based on this cohort, ADRs and genetic susceptibility in anti-TB treatment patients were widely studied, ${ }^{819-23}$ which made a great contribution to the pharmacoepidemiology of Chinese TB patients. However, two limitations cannot be ignored. First, blood samples were collected with Flinders Technology Associates $\left(\mathrm{FTA}^{\mathrm{TM}}\right.$, GE Whatman, Maidstone, Kent, United Kingdom) cards, which could not be used in subsequent high-throughput genomics studies and led to the inability to conduct serum marker studies. The combination of chemical, biological and large-scale observational health data is critical to predict ADRs in both individual patients and global populations. ${ }^{24}$ In addition, serum or plasma samples obtained both before and during treatment of all subjects are also needed to enable future studies of new proteomic, metabonomic and other soluble biomarkers or predictors of drug-induced hepatotoxicity. ${ }^{25}$ Second, only sputum smear-positive pulmonary TB patients treated with the DOTS strategy were included, and the percentage of bacteriologically confirmed cases among total new and relapsed pulmonary TB cases was only $32 \%$ in 2017. ${ }^{1}$ Thus, it is necessary to study ADRs in other types of patients. In addition to first-line drugs, the number of second-line drugs and new drugs for treating different types of DR-TB patients are also gradually increasing, ${ }^{12}$ and ADRs induced by those drugs still need further study. Thus far, there are no other similar new cohorts in China that can satisfy the need for a study of ADEs/ADRs.

For these reasons, we plan to establish a new prospective observational cohort, the Home-based Anti-Tuberculosis Treatment Adverse Reactions (HATTAR) study, based on newly diagnosed TB patients receiving homebased anti-TB treatment in China, and to recognise the incidences, prognosis and risk factors of anti-TB drug-induced ADRs in real-world experiences. The goals are as follows:

1. To complete an epidemiological survey of anti-TB treatment patients, including baseline characteristics, diagnosis and treatment, clinical outcomes, ADEs/ ADRs, risk factors and to evaluate the incidence of various ADEs/ADRs in this population.

2. To build a biological specimen bank for future research, including plasma and blood cells at different treatment times and incorporating existing routine biochemical test results.

3. To explore demographic factors, genetic variants and environmental risk factors relevant to anti-TB induced ADEs/ADRs, and their interactions.

\section{METHODS AND ANALYSIS}

\section{Study design}

This is a multicentre, prospective observational cohort study of Chinese patients being treated for TB, sponsored by Nanjing Medical University. The participating hospitals are two infectious disease hospitals and two local designated TB diagnosis and treatment centres in general hospitals located in Jiangsu Province of China.

\section{Study population}

Recruitment of a baseline cohort of 3200 newly diagnosed TB patients will be conducted between January 2019 and December 2020. The patients will be followed up until finishing anti-TB treatment.

Newly diagnosed TB patients who are ready to receive anti-TB treatment, are willing to join the study and sign the informed consent form or have a surrogate do so will be included in this study.

Exclusion criteria: (1) having a psychiatric disease and unable to fill out the self-recorded diaries during the anti-TB treatment and (2) having a severe disease with a life-expectancy shorter than 6 months. 


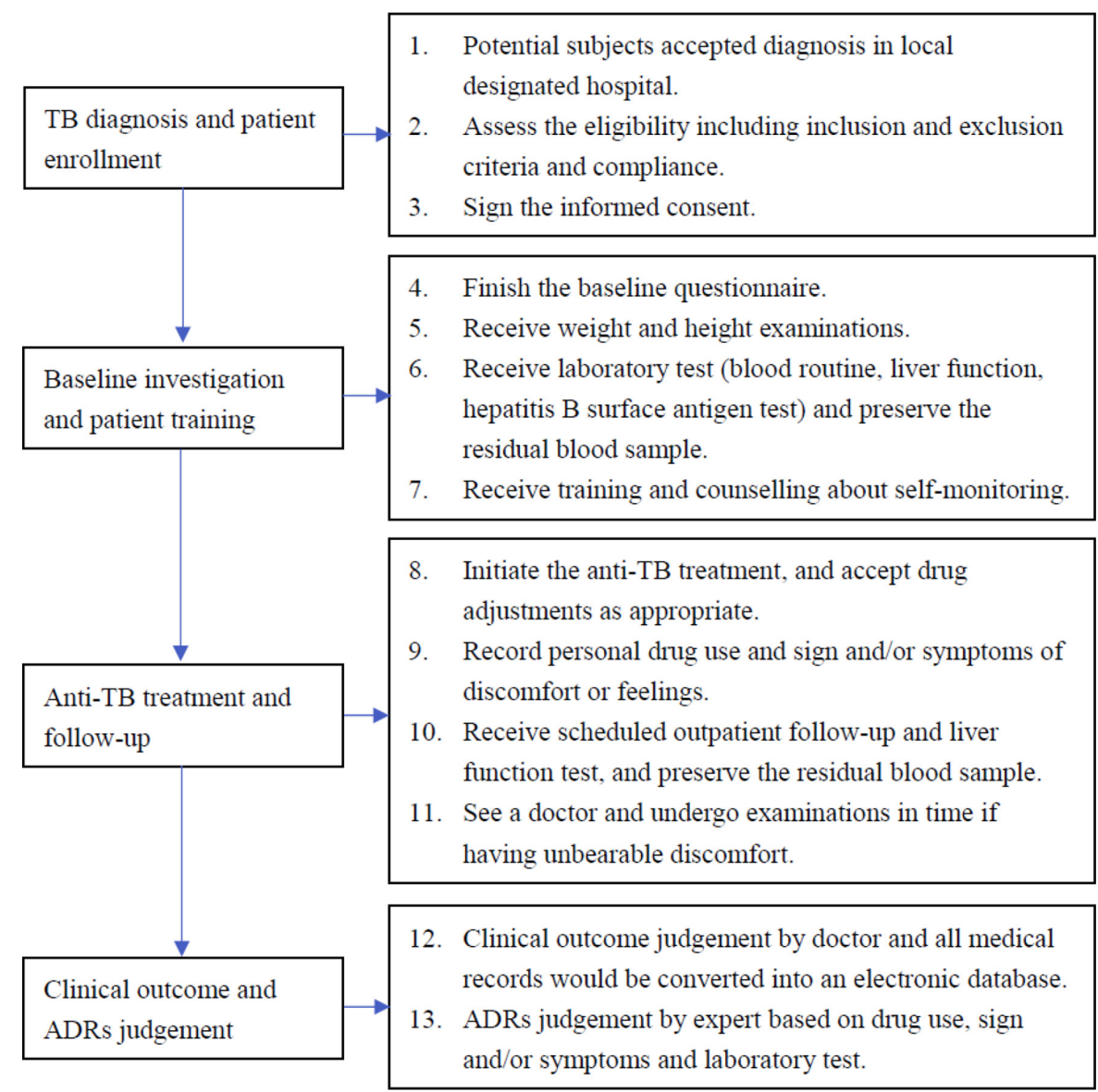

Figure 1 Flowchart of study design and procedures. ADRs, adverse drug reactions; TB, tuberculosis.

Withdrawal criteria: (1) unwilling to continue participating in the study; (2) loss to follow-up (stopping the drugs for longer than 2 months); (3) developing serious diseases that prevent them from continuing anti-TB treatment; (4) moving or travelling and thus missing all the scheduled laboratory tests in the first 2 months and (5) death that is not caused by TB or anti-TB drug-induced ADRs.

\section{Study protocol}

This study protocol was based on the National Tuberculosis Prevention and Control Planning and the Guideline for TB Diagnosis and Treatment in China, ${ }^{26}$ which consists of four main steps: TB diagnosis and patient enrollment, baseline investigation and patient training, anti-TB treatment and follow-up, clinical outcome and ADRs classification. A flowchart of the study design and procedures is shown in figure 1 .

\section{TB diagnosis and patient enrollment}

Potential subjects will be recruited from Zhenjiang, Changshu, Taixing and Jurong between January 2019 and December 2020 and diagnosed based on Chinese national TB diagnostic guidelines (WS288-2017), which rely on clinical judgement based on symptoms, chest radiography, tuberculin skin tests and smear microscopy.

If newly diagnosed TB patients meet the inclusion criteria, the doctors will explain the study to the patients and invite them to participate. If the patients agree, informed consent will be signed by the patient or a surrogate.

\section{Baseline investigation and patient training}

TB patients who have been recruited will complete the baseline questionnaire, including personal history of diseases and smoking and drinking habits, and undergo anthropometric measurements (weight and height). The patients will also be subjected to blood collection and laboratory tests, including routine blood tests and liver function and hepatitis B surface antigen tests. The residual blood sample of each patient will be separated into plasma and blood cells according to the standard laboratory protocol and then immediately frozen at $-40^{\circ} \mathrm{C}$.

The enrolled TB patients will receive training and counselling about medication knowledge and self-monitoring knowledge, for example, the importance of scheduled liver function tests, how to fill out the self-recorded diaries, what uncomfortable symptoms need to be recorded, and how to get consulting services.

\section{Anti-TB treatment and follow-up}

Once diagnosed with $\mathrm{TB}$, patients will begin to receive standardised anti-TB treatment according to the WHO guidelines for the treatment of drug-susceptible and drug-resistant TB. ${ }^{27} 28$ For example, in patients with 
drug-susceptible pulmonary TB, isoniazid (INH), rifampicin (RIF), pyrazinamide (PZA) and ethambutol (EMB) will be given for 2 months in the intensive phase, and then, INH and RIF, for the 4 months of the continuation phase. The doctors can decide whether hospitalisation admission is needed according to the patient's condition. Inpatients after discharge and other patients who do not need hospitalisation admission will receive community- or home-based DOTS regimens. The doctors can also change or adjust the medication according to the patient's condition during the treatment period.

After initiating the anti-TB treatment, all patients will be followed up until finishing treatment unless they withdraw. Patients will be asked to record personal drug use and signs and/or symptoms of discomfort or unfavourable feelings in the self-recorded diaries. During the first 2 months, patients will receive scheduled outpatient follow-up and liver function tests every 2 weeks in the designated hospital according to the local free-TB service policy. If patients exhibit unbearable discomfort or some symptoms of suspected hepatitis (such as anorexia, nausea, vomiting, malaise or tea-coloured urine) or other diseases, they should see a doctor and undergo examinations. After the first 2 months, patients can volunteer to go to the hospital for medical examinations.

During the treatment, if suspicious ADEs emerge, the local supervising doctors will conduct investigations to rule out other possible causes and fill out the relevant questionnaire. If the scheduled laboratory tests of the patients without any discomfort show an abnormality, the ADE investigation procedure will also be activated. Furthermore, the local supervising doctors will be required to actively contact the patients and check their self-recorded diaries for signs and/or symptoms of ADEs/ ADRs and drug use. Additionally, the residual blood sample of each patient after the scheduled laboratory tests will be preserved and frozen at $-40^{\circ} \mathrm{C}$.

Every TB patient in Jiangsu Province has a special outpatient medical record, which is used to record all of the treatment behaviours of patients throughout the treatment period, including basic personal information, diagnosis, medication history, laboratory test and suspicious ADEs/ADRs. In addition, every local supervising doctor will be provided with a registration and management card for every TB patient, which will be used to record basic personal information, patient management methods and treatment outcomes. Both the medical record and the management card are based on the patient's China ID number.

\section{Clinical outcome and ADR classification}

When all TB patients finish treatment, the local supervising doctors will comprehensively judge the patient's treatment outcomes according to the WHO definitions (cured, treatment completed, treatment failed, died, lost to follow-up, not evaluated, treatment success), and record them on the management card of every patient. The outpatient medical record and the management card will be collected and converted into an electronic database.

Based on medication records, symptoms, signs, laboratory test results, relevant investigations and self-recorded diaries, local supervising doctors will be able to judge ADEs preliminarily. Experts from the ADRs monitoring centre will regularly review and verify the judgements and classify all the ADEs into eight categories as follows ${ }^{829}$ : (1) liver dysfunction, including elevated transaminase and jaundice; (2) gastrointestinal reactions, including nausea, vomiting and diarrhoea; (3) drug allergy, including pruritus and rash; (4) arthralgia or muscle pain; (5) nervous system disorders, including sleep disorders, dizziness, headache, tinnitus and hearing loss; (6) haematological system disorders, including anaemia and leucopenia; (7) renal impairment, including decline of renal function, positive urine protein and renal failure and (8) cardiac injury, including QT prolongation; (9) others. Finally, the experts will conduct causality assessments based on the WHO-UMC causality assessment system $^{30}$ and the Roussel Uclaf Causality Assessment Method $^{31}$ and judge some ADEs as ADRs according to the criteria.

Anti-TB drug-induced hepatotoxicity (ATDH) is defined as (1) an increase in alanine aminotransferase (ALT) levels greater than two times the upper limit of normal (ULN) with/without a combined increase in aspartate aminotransferase (AST) and total bilirubin levels provided one of them was greater than two times the ULN during the treatment ${ }^{32}$ or (2) the causality assessment result is highly probable, probable or possible. The severity of hepatotoxicity is classified into mild, moderate and severe according to the WHO toxicity classification standards. ${ }^{33}$ The clinical types of hepatotoxicity are classified into hepatocellular, cholestatic or mixed types based on the ratio (R) of elevation of baseline ALT to baseline alkaline phosphatase. ${ }^{32}$

\section{Sample size calculation}

The sample size calculation was based on the genetic susceptibility study to be carried out in the future. We were interested in calculating the sample size needed for an effect size (ORs) of 1.8 with at least $90 \%$ power under the dominance model, where the minor allele frequency was set at $10 \%$, with a type I error level of 0.05 under a matched case-control study design. The incidence of ATDH in Chinese anti-TB treatment patients is $11.9 \%{ }^{18}$ The Quanto statistical programV.1.2.4 (University of Southern California, Los Angeles, USA) ${ }^{34}$ was used to calculate the sample size of a genetic susceptibility study for ATDH. The calculated sample size was 354 ATDH cases. This number of cases can be obtained from 2975 $(354 / 11.9 \%)$ TB patients who finished anti-TB treatment. Considering a withdrawal or loss of follow-up rate of $6 \%,{ }^{23}$ the calculated sample size was 3165 TB patients. Therefore, we set the target sample size to 3200 newly diagnosed TB patients. Based on our previous ADACS cohort, and setting the exclusion rate to $2.4 \%$ and the 
participation rate of eligible subjects to $71.2 \%,{ }^{23}$ at least 4600 newly diagnosed TB patients will be needed within 2 years. According to the number of newly diagnosed TB patients in each hospital per year (600 patients per hospital), the total number of TB patients in four hospitals is almost 2400 per year, which fully meets the sample size requirement in 2 years.

\section{Data analysis plan}

Continuous variables will be described as the mean $\pm \mathrm{SD}$ or as median with IQR, and differences between groups will be analysed by one-way analysis of variance or non-parametric tests. Blood biochemical indicators in different groups at different times will be compared using variance analysis of repeated measures data. Categorised variables will be expressed as numbers and percentages and analysed by $\chi^{2}$ tests or a logistic regression model. The incidence of different ADEs/ADRs will be reported by descriptive statistics, and subgroup analysis will be used to provide descriptive results for different groups of patients. Unmatched nested case-control study will be used to analyse the influencing factors on the incidence of ADRs (including ATDH). Patients who fulfilled the ATDH criteria will be assigned to the case group, whereas controls will be selected from those with sustained normal liver function through the whole therapy. Furthermore, matched nested case-control study will be used to explore the role of genetic variations (single nucleotide polymorphisms) in susceptibility to ATDH. For each ATDH case, two controls will be selected randomly and matched for the place of sample collection, age (within 5 years), sex and treatment history. Multivariate conditional logistic regression analysis will be used to estimate the association between various factors and the risk of ADRs by ORs and 95\% CIs, with potential confounders as covariates. The Cox proportional-hazards regression model will be used in the analysis of time-to-event data. Bonferroni correction method will be applied to adjust the $p$ value for multiple comparisons. All analyses will be performed using SPSS V.20.0 for Windows. A two-tailed $p$ value $<0.05$ will be considered significant.

\section{Patient and public involvement}

Patients or public were not involved in the development of the research question and study design or conducting the present study. All participants will be informed of the results through post or email.

\section{Ethics and dissemination}

Written informed consent will be signed by every patient or a surrogate before enrollment. Patients can withdraw from the study at any point with no effect on their clinical care. The patients' blood sample in this study will come from the residual blood samples after routine laboratory tests, which will not increase the amount of blood taken or the number of blood samples taken. All data will be kept anonymous and managed with confidentially. The findings of the study will be published in peer-reviewed journals and will be presented at national and international conferences.

\section{DISCUSSION}

China is one of the 20 highest TB-burdened countries, ${ }^{1}$ and in 2010, TB was more prevalent in rural areas $(163 / 100000)$ than urban ones $(73 / 100000),{ }^{35}$ which is one of the causes of unfavourable treatment outcomes. ${ }^{36}$ A previous study showed that patients from rural areas were less aware of ADRs than those from urban areas. ${ }^{37}$ Therefore, in the case of many rural TB patients in China, a key issue in treatment success is to improve their self-reporting rates of ADEs/ADRs. Furthermore, the potential of patient self-reporting has been described in the literature as a noteworthy source of new information about early detection of new, rare and serious ADRs. ${ }^{38}$ The information provided by patient reports can also be significant in ADR detection. ${ }^{39}$ However, patient self-reporting rates and awareness are still low. ${ }^{40}$ In recent years, many electronic methods have been used to increase reporting rates, especially in cancer treatment, ${ }^{41-43}$ but those methods are obviously inappropriate for Chinese rural patients in a developing country. In the present study, a method combining active self-recorded diaries and passive scheduled laboratory tests was used to enhance the identification of adverse reactions. In our previous ADACS cohort study, active self-recorded diaries were used in TB patients during treatment, which were simple, practical and easy to maintain. ${ }^{18}$ The local supervising doctors regularly checked and reviewed the patient records and timely identified some problems. In addition, the local free-TB service policy, especially free liver function tests in the first 2 months, also facilitates the timely detection of ADEs/ADRs. Most ADRs induced by anti-TB drugs occur within the first 2 months of treatment. ${ }^{8} 44$ Therefore, our approach may be a good way to identify ADEs/ADRs during home-based anti-TB treatment.

One of the purposes of ADR monitoring is to identify risk factors that may predispose, induce or influence the development, severity and incidence of adverse reactions in population samples, such as genetics, racial differences, diets, diseases, prescribing practices, cultures of drug use and traditions of the people. ${ }^{45}$ Our study will not only evaluate the incidence of ADEs/ADRs but will also collect samples for future research on risk factors for ADEs/ADRs, such as genetic polymorphisms. The sample size calculation of this protocol was based on studies of genetic analyses of ATDH. In previous studies, the sample sizes used have been relatively small (ranging from $8^{46}$ to $461^{47}$ ), which makes it difficult to achieve an adequate statistical power. Furthermore, too small a sample size to detect true evidence for an association increases false negative rates and reduces the reliability of a study, ${ }^{48}$ which is one of the reasons for heterogeneity of results between different studies. Additionally, plasma specimens collected at different time points during treatment from each patient are also important for ADR studies. 
Previous studies have shown that oxidative stress, and more broadly, disturbances in redox homeostasis alongside mitochondrial dysfunction, may contribute to the hepatotoxicity induced by first-line anti-TB drugs ${ }^{49} \mathrm{~A}$ rat study suggested that INH may initiate its toxicity in liver mitochondria through interactions with electron transfer chains, lipid peroxidation, mitochondrial membrane potential decline and cytochrome c expulsion, which ultimately leads to cell death. ${ }^{50}$ The activity of glutathione in ATDH patients is reduced and the level of malondialdehyde is increased. ${ }^{51}$ In addition, plasma specimens could be used to study the relationships among the changes in blood drug concentration, oxidative stress index and ADRs, which facilitate the study of mechanisms from the perspective of the population.

The HATTAR study will be conducted similarly to our previous population-based cohort study, which provides a good technical basis for us to carry out some new research. However, there are still two limitations in our study. One limitation is that we will recruit the potential eligible patients, including the floating population (namely, people who engage in partial temporary relocation, whose registration of legal residence remains in their original place of habitation and who are ineligible for permanent residence in the locale into which they moved $^{52}$ ), who will add uncertainty to the follow-up. The floating population is a high-risk group not only for TB infection $^{53}$ but also for treatment failure or drug resistance. ${ }^{54}$ Although the risk of being lost to follow-up of this population is high, risk factor studies of ADRs in this population are still helpful in improving treatment adherence. In addition, considering safety and practical necessity, rechallenge with the drug suspected of causing the ADRs will not be done in this study. Furthermore, among the first line anti-TB drugs prescribed, INH, RIF and PZA are potentially hepatotoxic. ${ }^{33}$ Under the combination therapy strategy, it is difficult to judge which drug or drug combination is the main causative agent leading to ADRs.

\section{Author affiliations \\ ${ }^{1}$ Department of Epidemiology, School of Public Health, Nanjing Medical University, Nanjing, Jiangsu, China \\ ${ }^{2}$ Department of Tuberculosis, The Third People's Hospital of Zhenjiang affiliated to Jiangsu University, Zhenjiang, China \\ ${ }^{3}$ Department of Tuberculosis, The Second People's Hospital of Changshu, Changshu, China \\ ${ }^{4}$ Department of Infectious Disease, The People's Hospital of Taixing, Taixing, China ${ }^{5}$ Department of Infectious Disease, The Jurong Hospital Affiliated to Jiangsu University, Jurong, China \\ ${ }^{6}$ Department of Biostatistics, School of Public Health, Nanjing Medical University, Nanjing, Jiangsu, China}

Contributors ST and HP obtained the research funding and is the principal investigator of the study. MY, HP and LL conceived and designed this study. MY and HP drafted the manuscript. XH, HC, BT, WL, HY and ST all participated in the final design of the study and revisions of this manuscript.

Funding This work was supported by National Natural Science Foundation of China (81373066), Priority Academic Program Development of Jiangsu Higher Education Institutions (PAPD) and Key Research and Development Plan of Zhenjiang (SHW2016008, SH2017022).
Competing interests None declared.

Patient consent for publication Obtained.

Ethics approval The ethics committees of Nanjing Medical University (NMU 2018-579).

Provenance and peer review Not commissioned; externally peer reviewed.

Open access This is an open access article distributed in accordance with the Creative Commons Attribution Non Commercial (CC BY-NC 4.0) license, which permits others to distribute, remix, adapt, build upon this work non-commercially, and license their derivative works on different terms, provided the original work is properly cited, appropriate credit is given, any changes made indicated, and the use is non-commercial. See: http://creativecommons.org/licenses/by-nc/4.0/.

\section{REFERENCES}

1. WHO. Global tuberculosis report 2018. Geneva: World Health Organization, 2018.

2. WHO. Global tuberculosis control: surveillance, planning and Financing. Geneva: World Health Organization, 2005.

3. Ali MK, Karanja S, Karama M. Factors associated with tuberculosis treatment outcomes among tuberculosis patients attending tuberculosis treatment centres in 2016-2017 in Mogadishu, Somalia. Pan Afr Med J 2017;28:197.

4. Michael OS, Sogaolu OM, Fehintola FA, et al. Adverse events to first line anti-tuberculosis drugs in patients co-infected with HIV and tuberculosis. Ann Ib Postgrad Med 2016;14:21-9.

5. Nebeker JR, Barach P, Samore MH. Clarifying adverse drug events: a clinician's guide to terminology, documentation, and reporting. Ann Intern Med 2004;140:795-801.

6. Kalaiselvan V, Kumar P, Mishra P, et al. System of adverse drug reactions reporting: What, where, how, and whom to report? Indian $J$ Crit Care Med 2015;19:564-6.

7. Castro AT, Mendes M, Freitas S, et al. Incidence and risk factors of major toxicity associated to first-line antituberculosis drugs for latent and active tuberculosis during a period of 10 years. Rev Port Pneumol 2015;21:144-50.

8. Lv X, Tang S, Xia Y, et al. Adverse reactions due to directly observed treatment strategy therapy in Chinese tuberculosis patients: a prospective study. PLoS One 2013;8:e65037.

9. Zhang Y, Wu S, Xia Y, et al. Adverse events associated with treatment of multidrug-resistant tuberculosis in China: an ambispective cohort study. Med Sci Monit 2017;23:2348-56.

10. Schnippel K, Berhanu RH, Black A, et al. Severe adverse events during second-line tuberculosis treatment in the context of high HIV Co-infection in South Africa: a retrospective cohort study. BMC Infect Dis 2016;16:593.

11. Lin FS, Wu MY, Tu WJ, et al. A cross-sectional and follow-up study of leukopenia in tuberculosis patients: prevalence, risk factors and impact of anti-tuberculosis treatment. J Thorac Dis 2015;7:2234-42.

12. Tiberi S, Buchanan R, Caminero JA, et al. The challenge of the new tuberculosis drugs. La Presse Médicale 2017;46:e41-e51.

13. Vasava MS, Bhoi MN, Rathwa SK, et al. Drug development against tuberculosis: Past, present and future. Indian J Tuberc 2017;64:252-75.

14. Cardoso MA, do Brasil P, Schmaltz CAS, et al. Tuberculosis Treatment Outcomes and Factors Associated with Each of Them in a Cohort Followed Up between 2010 and 2014. Biomed Res Int 2017;2017:1-7.

15. Naik BR, Shastri SG, Vishwanath NS, et al. Adverse drug reactions in tuberculosis patients: consequences for TB control. Int J Tuberc Lung Dis 2015;19:621-2.

16. Loveday M, Wallengren $\mathrm{K}$, Brust J, et al. Community-based care vs. centralised hospitalisation for MDR-TB patients, KwaZulu-Natal, South Africa. Int J Tuberc Lung Dis 2015;19:163-71.

17. Taneja N, Chellaiyan VG, Daral S, et al. Home based care as an approach to improve the efficiency of treatment for MDR tuberculosis: a Quasi-Experimental Pilot Study. J Clin Diagn Res 2017;11:Lc05-Lc08.

18. Xia YY, Hu DY, Liu FY, et al. Design of the anti-tuberculosis drugs induced adverse reactions in China National Tuberculosis Prevention and Control Scheme Study (ADACS). BMC Public Health 2010;10:267.

19. Tang SW, Lv XZ, Zhang Y, et al. CYP2E1, GSTM1 and GSTT1 genetic polymorphisms and susceptibility to antituberculosis drug-induced hepatotoxicity: a nested case-control study. J Clin Pharm Ther 2012;37:588-93. 
20. Shang P, Xia Y, Liu F, et al. Incidence, clinical features and impact on anti-tuberculosis treatment of anti-tuberculosis drug induced liver injury (ATLI) in China. PLoS One 2011;6:e21836.

21. Tang SW, Lv XZ, Chen R, et al. Lack of association between genetic polymorphisms of CYP3A4, CYP2C9 and CYP2C19 and antituberculosis drug-induced liver injury in a community-based Chinese population. Clin Exp Pharmacol Physiol 2013;40:326-32.

22. Chen R, Wang J, Tang SW, et al. CYP7A1, BAAT and UGT1A1 polymorphisms and susceptibility to anti-tuberculosis drug-induced hepatotoxicity. Int J Tuberc Lung Dis 2016;20:812-8.

23. Wu S, Xia Y, Lv X, et al. Preventive use of hepatoprotectors yields limited efficacy on the liver toxicity of anti-tuberculosis agents in a large cohort of Chinese patients. J Gastroenterol Hepatol 2015;30:540-5.

24. Boland MR, Jacunski A, Lorberbaum T, et al. Systems biology approaches for identifying adverse drug reactions and elucidating their underlying biological mechanisms. Wiley Interdiscip Rev Syst Biol Med 2016;8:104-22.

25. Avigan Ml, Bjornsson ES, Pasanen M, et al. Liver safety assessment: required data elements and best practices for data collection and standardization in clinical trials. Drug Saf 2014;37(Suppl 1):S19-S31.

26. Wu S, Wang H, Li B. Tuberculosis controlling, from China's perspective. J Infect Public Health 2018;11:300.

27. WHO. Guidelines for treatment of drug-susceptible tuberculosis and patient care, 2017 update 2017.

28. WHO. WHO treatment guidelines for drug-resistant tuberculosis, 2016 update, 2016.

29. Zhang T, Du J, Yin X, et al. Adverse events in treating smear-positive tuberculosis patients in China. Int $J$ Environ Res Public Health 2015;13:86.

30. WHO. The use of the WHO-UMC system for standardized case causality assessment. Uppsala: The Uppsala Monitoring, 2005.

31. Aithal GP, Watkins PB, Andrade RJ, et al. Case definition and phenotype standardization in drug-induced liver injury. Clin Pharmacol Ther 2011;89:806-15.

32. Bénichou C. Criteria of drug-induced liver disorders. Report of an international consensus meeting. J Hepatol 1990;11:272-6.

33. Tostmann A, Boeree MJ, Aarnoutse RE, et al. Antituberculosis druginduced hepatotoxicity: concise up-to-date review. J Gastroenterol Hepatol 2008;23:192-202.

34. Wj G, Jm M. QUANTO 1.1: a computer program for power and sample size calculations for genetic-epidemiology studies. 2006. http://biostats.usc.edu/software

35. Wang $\mathrm{L}$, Zhang $\mathrm{H}$, Ruan $\mathrm{Y}$, et al. Tuberculosis prevalence in China, 1990-2010; a longitudinal analysis of national survey data. Lancet 2014;383:2057-64.

36. Lin Y, Enarson DA, Du J, et al. Risk factors for unfavourable treatment outcome among new smear-positive pulmonary tuberculosis cases in China. Public Health Action 2017;7:299-303.

37. Joshi A, Shah N, Mistry M, et al. Evaluation of knowledge and perception toward adverse drug reactions among patients visiting tertiary-care teaching hospital. Natl J Physiol Pharm Pharmacol 2015;5:280.
38. Inácio $\mathrm{P}$, Cavaco A, Airaksinen M. The value of patient reporting to the pharmacovigilance system: a systematic review. $\mathrm{Br} J$ Clin Pharmacol 2017;83:227-46.

39. Hazell L, Cornelius V, Hannaford P, et al. How do patients contribute to signal detection?: a retrospective analysis of spontaneous reporting of adverse drug reactions in the UK's Yellow Card Scheme. Drug Saf 2013;36:199-206.

40. Margraff $F$, Bertram $D$. Adverse drug reaction reporting by patients: an overview of fifty countries. Drug Saf 2014;37:409-19.

41. Davoodi S, Mohammadzadeh Z, Safdari R. Mobile Phone Based System Opportunities to Home-based Managing of Chemotherapy Side Effects. Acta Inform Med 2016;24:193-6.

42. Holch P, Warrington L, Bamforth LCA, et al. Development of an integrated electronic platform for patient self-report and management of adverse events during cancer treatment. Ann Oncol 2017;28:2305-11.

43. Holch P, Pini S, Henry AM, et al. eRAPID electronic patient selfReporting of Adverse-events: Patient Information and aDvice: a pilot study protocol in pelvic radiotherapy. Pilot Feasibility Stud 2018;4.

44. Arbex MA, Varella MC, Siqueira HR, et al. Antituberculosis drugs: drug interactions, adverse effects, and use in special situations. Part 1: first-line drugs. J Bras Pneumol 2010;36:626-40.

45. Srivastava Ak. Pharmacology: A Book Of Achieving Knowledge For Drugs. New Delhi: Educreation Publishing, 2017.

46. Roy B, Ghosh SK, Sutradhar D, et al. Predisposition of antituberculosis drug induced hepatotoxicity by cytochrome P450 2E1 genotype and haplotype in pediatric patients. J Gastroenterol Hepatol 2006;21:784-6.

47. Sun Q, Liu HP, Zheng RJ, et al. Genetic polymorphisms of SLCO1B1, CYP2E1 and UGT1A1 and susceptibility to anti-tuberculosis druginduced hepatotoxicity: a Chinese population-based prospective case-control study. Clin Drug Investig 2017;37:1125-36.

48. Hong EP, Park JW. Sample size and statistical power calculation in genetic association studies. Genomics Inform 2012;10:117-22.

49. Yew WW, Chang KC, Chan DP. Oxidative stress and first-line antituberculosis drug-induced hepatotoxicity. Antimicrob Agents Chemother 2018;62.

50. Ahadpour M, Eskandari MR, Mashayekhi V, et al. Mitochondrial oxidative stress and dysfunction induced by isoniazid: study on isolated rat liver and brain mitochondria. Drug Chem Toxicol 2016;39:224-32.

51. Chowdhury A, Santra A, Kundu S, et al. Induction of oxidative stress in antitubercular drug-induced hepatotoxicity. Indian J Gastroenterol 2001;20:97-100.

52. Chang S. The floating population: an informal process of urbanisation in China. Int J Popul Geogr 1996;2:197-214.

53. Li X, Li T, Tan S. Males, ages $\geq 45$ years, businessperson, floating population, and rural residents may be considered high-risk groups for tuberculosis infection in Guangzhou, China: a review of 136,394 tb confirmed cases. Rev Inst Med Trop Sao Paulo 2013;55:366-8.

54. Wang W, Wang J, Zhao Q, et al. Contribution of rural-to-urban migration in the prevalence of drug resistant tuberculosis in China. Eur J Clin Microbiol Infect Dis 2011;30:581-6. 